\title{
Marginal and Internal Adaptation Evaluation of Dental Composites Using Micro-CT
}

\author{
Adrian Tudor Stan, ${ }^{1}$ Laura Cîrligeriu $\left(\mathbb{D},{ }^{2}\right.$ Laura Idoraşi $\left(\mathbb{D},{ }^{1}\right.$ Meda Lavinia Negruţiu, ${ }^{1}$ \\ Cosmin Sinescu, ${ }^{1}$ Daniela Maria Pop, ${ }^{1}$ Tareq Hajaj, ${ }^{1}$ Şerban Talpoş, ${ }^{3}$ and Mihai Romînu ${ }^{1}$ \\ ${ }^{1}$ Victor Babes University of Medicine and Pharmacy, Faculty of Dental Medicine, \\ Department of Prosthesis Technology and Dental Materials, 2 Eftimie Murgu Sq., 300041 Timisoara, Romania \\ ${ }^{2}$ Victor Babes University of Medicine and Pharmacy, Faculty of Dental Medicine, \\ Department of Operative Dentistry and Endodontics, 2 Eftimie Murgu Sq., 300041 Timisoara, Romania \\ ${ }^{3}$ Victor Babes University of Medicine and Pharmacy, Faculty of Dental Medicine, Department of Oral and Maxillofacial Surgery, \\ 2 Eftimie Murgu Sq., 300041 Timisoara, Romania
}

Correspondence should be addressed to Laura Cîrligeriu; lauracirligeriu@gmail.com

Received 3 June 2019; Revised 26 August 2019; Accepted 11 September 2019; Published 13 October 2019

Guest Editor: Jung-Hwan Lee

Copyright (c) 2019 Adrian Tudor Stan et al. This is an open access article distributed under the Creative Commons Attribution License, which permits unrestricted use, distribution, and reproduction in any medium, provided the original work is properly cited.

\begin{abstract}
The aim of the study was to determine the marginal and internal adaptation after curing of different composite resin restorations, using a nondestructive X-ray micro-computed tomography (micro-CT). Forty previously extracted human molars, kept in $10 \%$ formalin to preserve the bonding capabilities of the tooth structures, were divided into four groups based on the composite system used and different light-curing times. Class II (vertical slot) cavities were prepared on one proximal side and restored with dental composite using a Tofflemire matrix, with a bulk-fill composite resin (Venus Bulk Fill, Heraeus Kulzer) and a universal posterior composite (G-ænial Posterior, GC). A curing lamp (Kerr Demi Ultra) was used with different curing times. Micro-CT scanning was performed by using Nikon XTH 225ST to reveal any defects in adaptation or gaps at the tooth restoration interface. The 3D images of the adaptation around the restorations were reconstructed using VG Studio Max 2.2 and myVGL 2.2.6 64-bit software. All samples from the G-ænial group showed marginal and internal gaps, with G-ænial Posterior having higher polymerization shrinkage and marginal gap values. In the Venus Bulk Fill group, there were fewer restorations with internal and external gap formation. Micro-CT is a three-dimensional imaging technique that can nondestructively detect adaptation around the resin composite restorations at every level of the sample.
\end{abstract}

\section{Introduction}

Present dental composites bearing inorganic components and an organic matrix are polymerized by quick reactions activated by, nowadays, a visible light. These dental restorative biomaterials are in constant and fast development, and thus preferred over amalgam or other alloys/metals for dental restorations [1].

They pose very good mechanical properties, but polymerization shrinkage and the stress developed and accumulated during shrinkage often lead to a lack of internal adaptation and gap formation at the tooth-restoration margin. These features possess considerable limitations of composite resin materials [2-4].

Controlling of the polymerization shrinkage stress developed by the composite resin material is critical to establish proper marginal integrity and, thus, the predictability and success of the restoration in the long-term. Internal and marginal gaps and lingering stresses notably affect the longevity of a restoration [5]. Therefore, it is fundamental to discern between the shrinkage mechanisms and develop new methods to bypass or prevent the unfavorable effects. The polymerization chain reaction of a light-cured bulk-fill composite resin differs with depth. The discrepancy is a 
result of progressive loss of penetrating power more profound inside the resin, conducting to a moderate polymerization reaction in the deeper layers [6-9].

The system reaction of dental composite light-curing develops in 3 main stages: pregel, gel point, and postgel. In the pre-gel phase, the resin can flow and go through molecular rearrangement in order to overcome shrinkage forces. During this stage, linear polymer chains are abundant and afterward, the resin goes from the flowable phase (pregel) to the viscous phase (postgel), which enacts its gel point. In the viscous stage, the resin displays a great modulus of elasticity, loses the flow capacity, and passes on the stress generated by polymerization contraction to the interface between tooth and restoration [10-12].

Research has determined that a different curing technique and time may impact the polymerization contraction of the dental composite $[13,14]$. The insufficient and partial curing of dental composites is correlated to a cutback in their mechanical properties and altered biocompatibility because of the elevated content of residual monomers and possible esthetic deficiencies, with an increased shift to staining of the resin surface and apparition of marginal gaps. Therefore, different curing techniques, like stepped, ramped, and pulsed-delay techniques have been proposed to overcome the issue related to polymerization contraction, specifically postoperative sensitivity, gap formation, and microleakage [11, 15].

Camargo et al. concluded that at the depth that corresponds to the resin increment recommended by the manufacturer $(2.0 \mathrm{~mm})$, all curing times and techniques gave adequate composite hardness, but below that level there is a possible lack or underpolymerized layer of composite which may lead to failures in adhesion [16, 17].

Recently, micro-computed tomography investigations are known as a reliable alternative to the traditional layer sectioning method for assessing internal and marginal adaptation and microleakage with different infiltration techniques. The rapid evolution of nondestructive tomography methods is important to foster further knowledge of polymerization contraction and microscopic details of the polymerization system for various dental resins. Furthermore, new advances in computer software have also facilitated the achievement of new understandings of composites polymerization using micro-CT [18-20]. Therefore, the aim of this study was to assess and evaluate the internal and marginal adaptation of two different composite resins with different curing times by the use of micro-CT technology.

\section{Materials and Methods}

Forty previously extracted human molars with no or minimal proximal cavities were selected for the present study. The dental calculus, remnants of periodontal tissue, and other tooth detritus were cleansed, and the teeth were stored in $10 \%$ formalin to preserve the bonding capabilities of the tooth structures [21]. The layout of the experimental groups was established with the following criteria: (1) restorative system and type of composite: bulk-fill composite resin (Venus Bulk Fill, Heraeus Kulzer) and a universal posterior hybrid composite (G-ænial Posterior, GC) and (2) different curing time
(20 seconds and 40 seconds). The groups are depicted in Table 1. Class II cavities (vertical slot) were prepared in the selected teeth by using a 245 pear-shaped diamond bur. The cavities were standardized with the following dimensions: width of $4 \mathrm{~mm}$, depth of $2 \mathrm{~mm}$, and height of $4 \mathrm{~mm}$, and they were prepared with the water cooling system activated on the mesial proximal tooth surface. The gingival margin was placed slightly occlusal from the cementoenamel junction (CEJ), and the cavities were prepared by a sole operator.

Afterward, the teeth were then randomly divided into 4 groups based upon the type of composite and curing time. An individual Tofflemire metallic matrix was placed on each tooth to reconstruct the proximal wall. The cavity was conditioned by using $37 \%$ phosphoric acid etching gel (Heraeus Kulzer) for 15 seconds and rinsed thoroughly with water. The excess moisture was removed by blowing with oilfree air. GLUMA Self Etch adhesive agent (one-step, selfetch from Heraeus Kulzer) and G-ænial Bond (one-step, self-etch 7th generation bonding agent from GC) were applied in the cavities and followed by 20 seconds light curing. Venus Bulk Fill A2 composite (Heraeus Kulzer) and G-ænial Posterior A2 (GC) were inserted into the cavities by using one bulk increment for the Venus Bulk Fill composite ( $4 \mathrm{~mm}$ thickness) and a $2 \mathrm{~mm}$ layering technique for the G-ænial Posterior, followed by different light polymerization in accordance to the prior specified groups (20 seconds or 40 seconds). The materials used in the study are characterized in Table 2. Visible light irradiation was performed, and the curing unit was a Demi ${ }^{\mathrm{TM}}$ Ultra Led Ultracapacitor Curing Light System (Kerr) with a light intensity of $1250 \mathrm{~mW} / \mathrm{cm}^{2}$, a wavelength of $450-470 \mathrm{~nm}$, and a light attachment of $8 \mathrm{~mm}$. The lamp possesses a fully integrated radiometer with a LED indicator that instantly communicates the curing light system's irradiance status. The polishing was made by using Composite Polishing Kit (Shofu); Dura-White stones for finishing, CompoSite polishers for prepolishing, and CompoSite Fine instruments for final polishing.

After the light-curing phase, the teeth were placed in a specimen holder and individually scanned by using micro$\mathrm{CT}$ at a resolution of $10 \mu \mathrm{m}$. Micro-CT scanning was performed by using the Nikon XTH 225 ST (Figure 1). The settings of the microfocus $\mathrm{X}$-ray source were as follows: $150 \mathrm{kV}, 100 \mu \mathrm{A}$, with the following detector: Pekin Elmer 1620 , 16-bit, $2000 \times 2000$ active pixels, $200 \mu \mathrm{m}$ pixel size, max frame rate $3.75 \mathrm{fps}$, microfocus source $-225 \mathrm{kV}$ rotating target option, and max power $450 \mathrm{~W}$ (focal spot size). The measurements and gap detections were made by the different coloring of the voxels made by the program varying with the size of the gaps.

The cross sections were acquired using VG Studio Max 2.2 (Figure 2) and myVGL 2.2.6 64-bit software. The data analysis was made by one evaluator in terms of marginal adaptation and measuring internal gap formation using SPSS mean and standard deviation.

\section{Results and Discussion}

The gap formation volume encompassing the restorations was measured as shown in Table 3. Considerable variations 
TABLE 1: Group descriptions.

\begin{tabular}{lcc}
\hline Group & Composite resin & Curing time (seconds) \\
\hline Group 1 & Venus Bulk Fill, Heraeus Kulzer & 20 \\
Group 2 & Venus Bulk Fill, Heraeus Kulzer & 40 \\
Group 3 & G-ænial Posterior, GC & 20 \\
Group 4 & G-ænial Posterior, GC & 40 \\
\hline
\end{tabular}

TABLE 2: Materials used in the present study.

\begin{tabular}{|c|c|c|c|}
\hline Material & Type & Composition & Description \\
\hline $\begin{array}{l}\text { Etching gel } \\
\text { (Heraeus Kulzer) }\end{array}$ & $37 \%$ phosphoric acid & $\begin{array}{l}37 \% \text { orthophosphoric acid, inorganic silica } \\
\text { gel thickener }\end{array}$ & Etching gel \\
\hline $\begin{array}{l}\text { Gluma self-etch } \\
\text { (Heraeus Kulzer) }\end{array}$ & Self-etch & $\begin{array}{c}\text { HEMA, acetone, maleic acid, TEGDMA, } \\
\text { UDMA, HEMA, Bis-GMA, filler, and } \\
\text { photoinitiator }\end{array}$ & $\begin{array}{l}\text { All-in-one bonding agent etches, primes, } \\
\text { bonds, and desensitizes in one single step }\end{array}$ \\
\hline G-ænial Bond & Self-etch & $\begin{array}{l}\text { Acetone, dimethacrylate, phosphoric acid } \\
\text { ester monomer, dimethacrylate component, } \\
\text { and photoinitiator }\end{array}$ & $\begin{array}{l}\text { HEMA free, one-step, self-etch } 7 \text { th } \\
\text { generation bonding agent }\end{array}$ \\
\hline $\begin{array}{l}\text { Venus Bulk Fill } \\
\text { (Heraeus Kulzer) }\end{array}$ & $\begin{array}{l}\text { Flowable bulk-fill } \\
\text { composite }\end{array}$ & $\begin{array}{l}\text { UDMA, EBADMA, fluoroaluminosilicate } \\
\text { glass, } \mathrm{YbF} \text {, and } \mathrm{SiO}\end{array}$ & $\begin{array}{l}\text { Permits easy filling of cavities in increments } \\
\text { of up to } 4 \mathrm{~mm}\end{array}$ \\
\hline $\begin{array}{l}\text { G-ænial Posterior } \\
\text { (GC) }\end{array}$ & $\begin{array}{l}\text { Light-cured hybrid } \\
\text { composite }\end{array}$ & $\begin{array}{c}\text { UDMA, dimethacrylate comonomers, } \\
\text { fluoroaluminosilicate glass, and fumed } \\
\text { silica }\end{array}$ & $\begin{array}{l}\text { Allows the light-curing of layers up } \\
\qquad 2.5-3 \mathrm{~mm}\end{array}$ \\
\hline
\end{tabular}

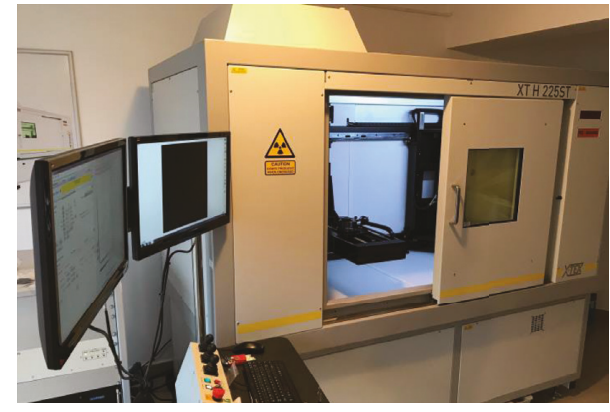

FIgure 1: The Nikon XTH 225 ST holder during the micro-CT scanning micro-computed tomography system.

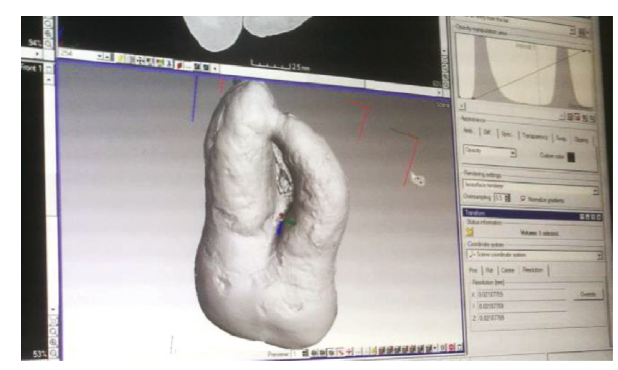

Figure 2: Analysis and reconstruction of the teeth using the VG Studio Max 2.2 software.

were detected between the different light-curing times, and differences were identified between the bulk-fill dental composite and the hybrid resin. Expanding the photoactivation time increased the apparition of marginal and internal gaps in all groups assessed. G-ænial groups displayed the highest mean in gap formation values.
TABle 3: Descriptive statistics of detected internal and marginal gaps and lack of adaptation. Means and standard deviations (SD) in $\mathrm{mm}^{3}$.

\begin{tabular}{lcccc}
\hline \multirow{2}{*}{ Group (sec) } & \multicolumn{2}{c}{ Internal } & \multicolumn{2}{c}{ Marginal } \\
& Mean & Std. deviation & Mean & Std. deviation \\
\hline G-ænial 20 & 1.52 & 0.67 & 0.38 & 0.30 \\
G-ænial 40 & 1.77 & 0.95 & 0.49 & 0.37 \\
Venus 20 & 0.31 & 0.27 & 0.14 & 0.15 \\
Venus 40 & 0.50 & 0.34 & 0.33 & 0.31 \\
\hline
\end{tabular}

The use of dental adhesive can be eliminated because the radiolucency of the adhesive would make it rather difficult to differentiate it from a gap in the micro-CT images, but clinically adhesive plays an important role in the formation of interfacial gaps and must, therefore, be included when internal and marginal gaps are being studied. Radiographic contrast agents could be used to display leakage and should be highly radiopaque in comparison to the composite material and tooth structures. The atomic number of silver is higher than that of the hard dental tissues and dental resin fillings; thus, silver nitrate is capable of presenting a good radiopaque contrast when it is dense. Further, the silver nitrate method of measuring microleakage is an acceptable technique because the silver ion is extremely small $(0.059 \mathrm{~nm})$ compared to the size of a typical bacterium $(0.5-1.0 \mu \mathrm{m})$, thereby making it more penetrative. The silver ion could be trapped in the dentinal tubules during the course of infiltration [22].

In the present study, gap formation was calculated by the volume between the delaminated composite and the walls of the tooth, internally, and the degree of volume that has a direct link with the external tooth-restoration interface, 


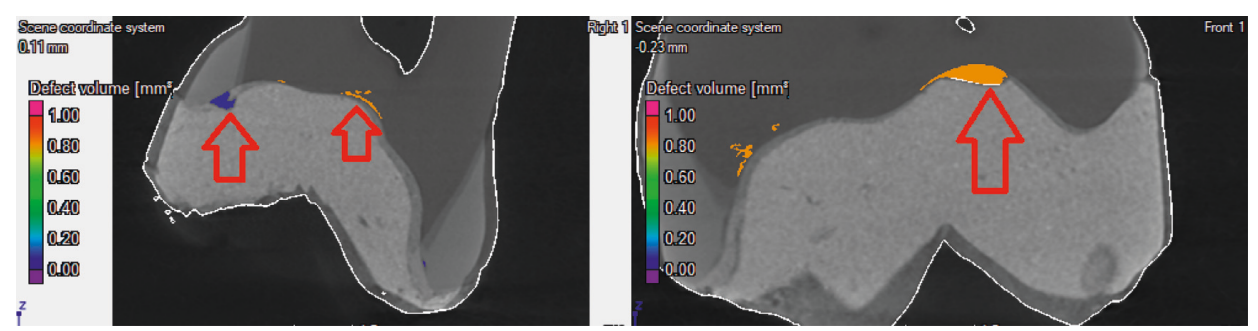

Figure 3: Typical micro-CT images of G-ænial Posterior groups indicating (red arrows) internal gaps.

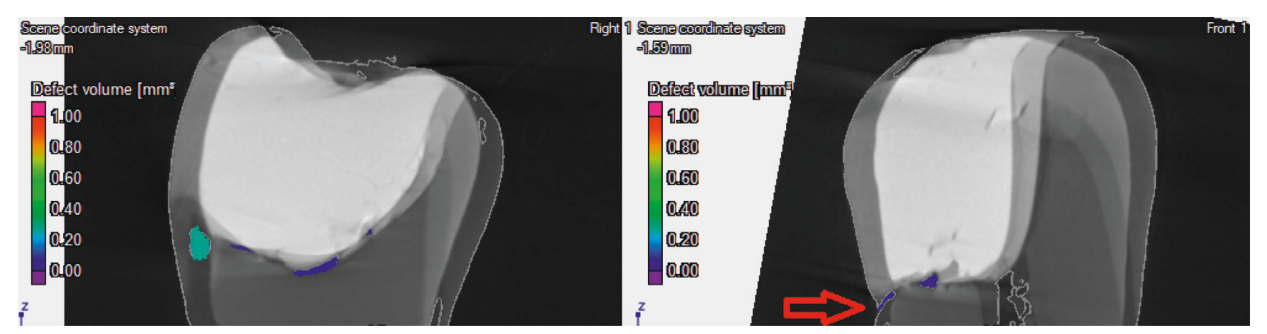

Figure 4: Typical micro-CT images of Venus Bulk Fill groups indicating (red arrow) marginal gaps.

marginally. However, some other studies have generally evaluated the microleakage at the external tooth-composite interface rather than internal adaptation [23].

Another aspect to be considered is that an appropriate analysis of the vast 3D data sets of scans and subsequent gaps was needed in order to provide a correct and valid interpretation. Considering the cavity shape, error possibility, and various factors that were taking part in the study, we analyzed only the marginal and internal adaptation, without assessing the possibility of marginal leakage using dye penetration technique, as the dye does not have the possibility of filling the internal gaps [24].

The most representative image sections are presented (Figures 3 and 4).

The results can be explained by the higher exposure time that enables greater internal stress because of the shrinkage of the restoration resins. On the other hand, a reduced level of exposure causes the reaction to occur in a slower manner and fewer polymer centers will develop. This slower reaction makes sure that a more linear polymeric structure is formed in the dental composite $[14,25,26]$. In this way, lower irradiance will partially suppress the boost in the viscosity of the polymer in the course of hardening and reduce the polymerization shrinkage and stress. A notable rise in gap formation and microleakage was noticed as the polymerization time was increased, and the marginal leakage and exposure time developed in a direct proportion.

Bouschlicher et al. also showed that the exposure time was important for determining the polymerization shrinkage force $[27,28]$. Therefore, light exposure for a 20 second period appears to produce appropriate polymerization as well as reducing the microleakage to a minimum.

In the present study, two composite materials were used for evaluating marginal leakage with two different curing times (20 and 40 seconds). The manufacturer recommends that Venus Bulk Fill be placed in $4 \mathrm{~mm}$ increments and thus, eliminating the need for time-consuming, $2 \mathrm{~mm}$ layering techniques, with light exposure of 20 seconds.

The evaluation of the level of marginal and internal adaptation goes hand in hand also with the length of the prepared cavity which can be measured prior in all dimensions. This can be demanding by the fact that the cavity line angles were round by the use of the 245 pear-shaped bur and not sharp with clear and distinct line angles and point angles.

Overall, micro-CT does not cause sample destruction and allows the detection of the deepest level of lack of adhesion and gap formation. These advantages result in a more accurate measurement of the bonding of the restorative materials in the tooth tissues.

\section{Conclusions}

Within the limitation of the present study, the following conclusions can be drawn: (a) the polymerization shrinkage was found to be intimately connected to the internal adaptation and marginal adaptation of the resin composite restorations; (b) considerable discrepancies were detected between the different polymerization times, and vast variations were identified between the bulk-fill dental composite and the hybrid resin, concluding that the bulk fill may be the better option; and (c) the proposed method for the evaluation of internal adaptation using a micro-CT system may provide a new measurement tool for assessing the internal adaptation of restorations by having a nondestructive approach.

\section{Data Availability}

The tables, figures, and scans used to support the findings of this study are available from the corresponding author upon request. 


\section{Conflicts of Interest}

The authors declare there are no conflicts of interest regarding the publication of this paper.

\section{Authors' Contributions}

All the authors have contributed equally to the manuscript and have approved its submission.

\section{Acknowledgments}

This work was supported, in part, by the Ph.D. grant of the Victor Babes University of Medicine and Pharmacy Timisoara $(3811 / 03.10 .2016)$.

\section{References}

[1] A. J. Feilzer, A. J. De Gee, and C. L. Davidson, "Curing contraction of composites and glass-ionomer cements," The Journal of Prosthetic Dentistry, vol. 59, no. 3, pp. 297-300, 1988.

[2] I. Sideridou, V. Tserki, and G. Papanastasiou, "Effect of chemical structure on degree of conversion in light-cured dimethacrylate-based dental resins," Biomaterials, vol. 23, no. 8, pp. 1819-1829, 2002.

[3] A. T. Stan, A. C. Cojocariu, A. A. Antal et al., "Marginal integrity evaluation of dental composite using optical coherence tomography," in High-End Medicine Based on Laser and Biotechnologies Proceedings of SPIE, D. C. Todea, A. G. Podoleanu, and V. F. Duma, Eds., vol. 9670, of Proceedings of the 5th Congress of the World-Federation-for-aserDentistry/6th International Conference on Lasers in Medicine, Article ID 967012, Romanian Soc Lasers Dentistry, Univ Med \& Pharmacy Victor Babes Timisoara, World Federat Laser Dentistry, Bucharest, Romania, May 2015.

[4] L. E. Cirligeriu, A. T. Stan, M. Rominu et al., "Evaluation of biodentine-tooth structures interfaces using laser scanning confocal microscopy," in Proceedings of the Seventh International Conference on Lasers in Medicine, D. C. Todea, A. G. Podoleanu, and V. F. Duma, Eds., vol. 10831, of 7th Romanian-Society-for-Laser-in-Dentistry (SRLS) International Conference on Lasers in Medicine/1st Congress of Laser in Dentistry, Article ID 108310Q, Romanian Soc Lasers Dent, Univ Med \& Pharmacy Victor Babes Timisoara, Timisoara, Romania, July 2017.

[5] E. Cho, A. Sadr, N. Inai, and J. Tagami, "Evaluation of resin composite polymerization by three dimensional micro-CT imaging and nanoindentation," Dental Materials, vol. 27, no. 11, pp. 1070-1078, 2011.

[6] A. T. Stan, A. Vaduva, M. L. Negrutiu et al., "Evaluation of dental composite resin interface and adhesion using laser scanning confocal microscopy," Materiale Plastice, vol. 55, no. 4, pp. 541-544, 2018.

[7] L. Rothmund, F.-X. Reichl, R. Hickel et al., "Effect of layer thickness on the elution of bulk-fill composite components," Dental Materials, vol. 33, no. 1, pp. 54-62, 2017.

[8] M. J. Roggendorf, N. Krämer, A. Appelt, M. Naumann, and R. Frankenberger, "Marginal quality of flowable 4-mm base vs. conventionally layered resin composite," Journal of Dentistry, vol. 39, no. 10, pp. 643-647, 2011.

[9] N. Ilie, A. Keßler, and J. Durner, "Influence of various irradiation processes on the mechanical properties and polymerisation kinetics of bulk-fill resin based composites," Journal of Dentistry, vol. 41, no. 8, pp. 695-702, 2013.

[10] C. L. Davidson and A. J. Feilzer, "Polymerization shrinkage and polymerization shrinkage stress in polymer-based restoratives," Journal of Dentistry, vol. 25, no. 6, pp. 435-440, 1997.

[11] R. L. Sakaguchi and H. X. Berge, "Reduced light energy density decreases post-gel contraction while maintaining degree of conversion in composites," Journal of Dentistry, vol. 26, no. 8, pp. 695-700, 1998.

[12] M. S. Soh and A. U. J. Yap, "Influence of curing modes on crosslink density in polymer structures," Journal of Dentistry, vol. 32, no. 4, pp. 321-326, 2004.

[13] M. R. Bouschlicher and F. A. Rueggeberg, "Effect of ramped light intensity on polymerization force and conversion in a photoactivated composite," Journal of Esthetic and Restorative Dentistry, vol. 12, no. 6, pp. 328-339, 2000.

[14] M. R. Bouschlicher, F. A. Rueggeberg, and D. B. Boyer, "Effect of stepped light intensity on polymerization force and conversion in a photoactivated composite," Journal of Esthetic and Restorative Dentistry, vol. 12, no. 1, pp. 23-32, 2000.

[15] B.-S. Lim, J. L. Ferracane, R. L. Sakaguchi, and J. R. Condon, "Reduction of polymerization contraction stress for dental composites by two-step light-activation," Dental Materials, vol. 18, no. 6, pp. 436-444, 2002.

[16] E. J. d. Camargo, E. Moreschi, W. Baseggio, J. A. Cury, and R. C. Pascotto, "Composite depth of cure using four polymerization techniques," Journal of Applied Oral Science, vol. 17, no. 5, pp. 446-450, 2009.

[17] M. F. Witzel, F. C. Calheiros, F. Gonçalves, Y. Kawano, and R. R. Braga, "Influence of photoactivation method on conversion, mechanical properties, degradation in ethanol and contraction stress of resin-based materials," Journal of Dentistry, vol. 33, no. 9, pp. 773-779, 2005.

[18] E.-J. Kim, K.-B. Lee, and M.-U. Jin, "Evaluation of marginal leakage of bulk fill flowable composite resin filling with different curing time using micro-computed tomography technology," Journal of Dental Rehabilitation and Applied Science, vol. 32, no. 3, pp. 184-193, 2016.

[19] R. Desantis, F. Mollica, D. Prisco, S. Rengo, L. Ambrosio, and L. Nicolais, "A 3D analysis of mechanically stressed dentin-adhesive-composite interfaces using X-ray microCT," Biomaterials, vol. 26, no. 3, pp. 257-270, 2005.

[20] J. Sun, N. Eidelman, and S. Lin-Gibson, "3D mapping of polymerization shrinkage using X-ray micro-computed tomography to predict microleakage," Dental Materials, vol. 25, no. 3, pp. 314-320, 2009.

[21] G. Tosun, Y. Sener, and A. Sengun, "Effect of storage duration/ solution on microshear bond strength of composite to enamel," Dental Materials Journal, vol. 26, no. 1, pp. 116-121, 2007.

[22] X. Zhao, S. Li, L. Gu, and Y. Li, "Detection of marginal leakage of class $\mathrm{V}$ restorations in vitro by micro-computed tomography," Operative Dentistry, vol. 39, no. 2, pp. 174-180, 2014.

[23] A. Peutzfeldt and E. Asmussen, "Determinants of in vitro gap formation of resin composites," Journal of Dentistry, vol. 32, no. 2, pp. 109-115, 2004.

[24] M. Atai and D. Watts, "A new kinetic model for the photopolymerization shrinkage-strain of dental composites and resin-monomers," Dental Materials, vol. 22, no. 8, pp. 785791, 2006.

[25] K.-D. Ahn, C.-M. Chung, and Y.-H. Kim, "Synthesis and photopolymerization of multifunctional methacrylates derived from Bis-GMA for dental applications," Journal of Applied Polymer Science, vol. 71, no. 12, pp. 2033-2037, 1999. 
[26] J. E. Klee, F. Neidhart, H.-J. Flammersheim, and R. Mülhaupt, "Monomers for low shrinking composites, 2. Synthesis of branched methacrylates and their application in dental composites," Macromolecular Chemistry and Physics, vol. 200, no. 3, pp. 517-523, 1999.

[27] M. R. Bouschlicher, M. A. Vargas, and D. B. Boyer, "Effect of composite type, light intensity, configuration factor, and laser polymerization on polymerization contraction forces," American Journal of Dentistry, vol. 10, no. 2, pp. 88-96, 1997.

[28] J. L. Ferracane, J. C. Mitchem, J. R. Condon, and R. Todd, "Wear and marginal breakdown of composites with various degrees of cure," Journal of Dental Research, vol. 76, no. 8, pp. 1508-1516, 1997. 


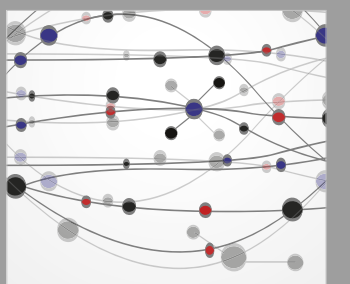

The Scientific World Journal
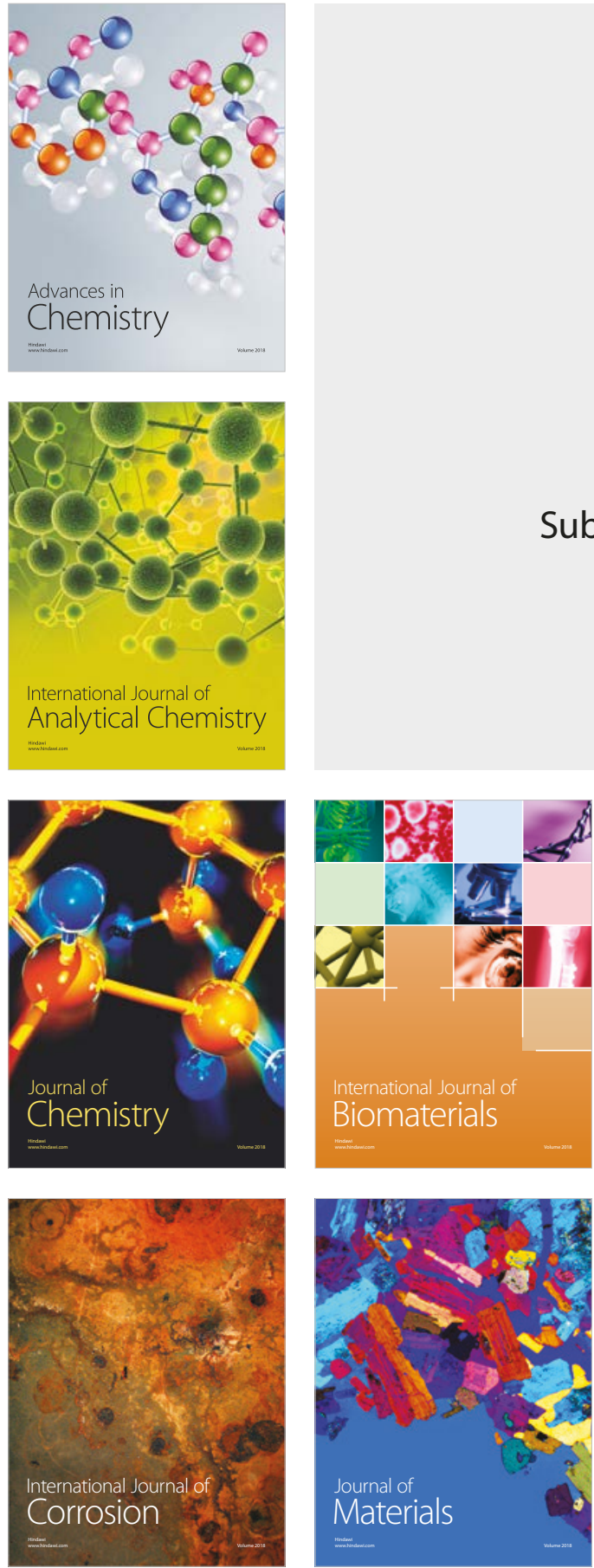

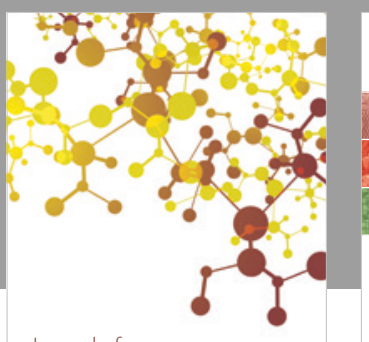

Journal of

Applied Chemistry
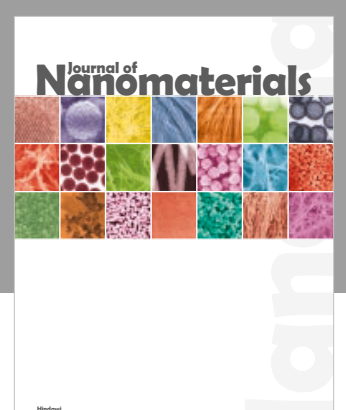

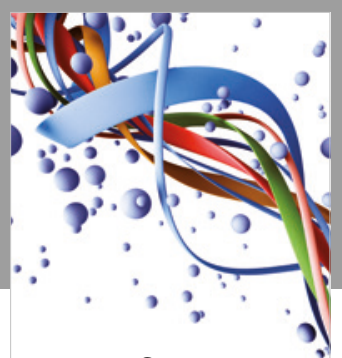

Scientifica

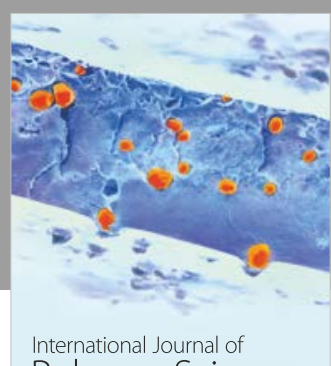

Polymer Science

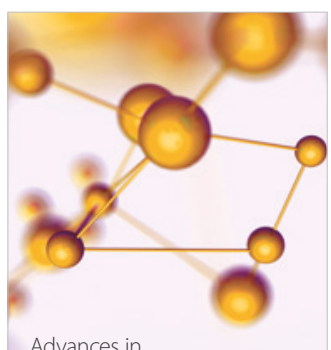

Physical Chemistry
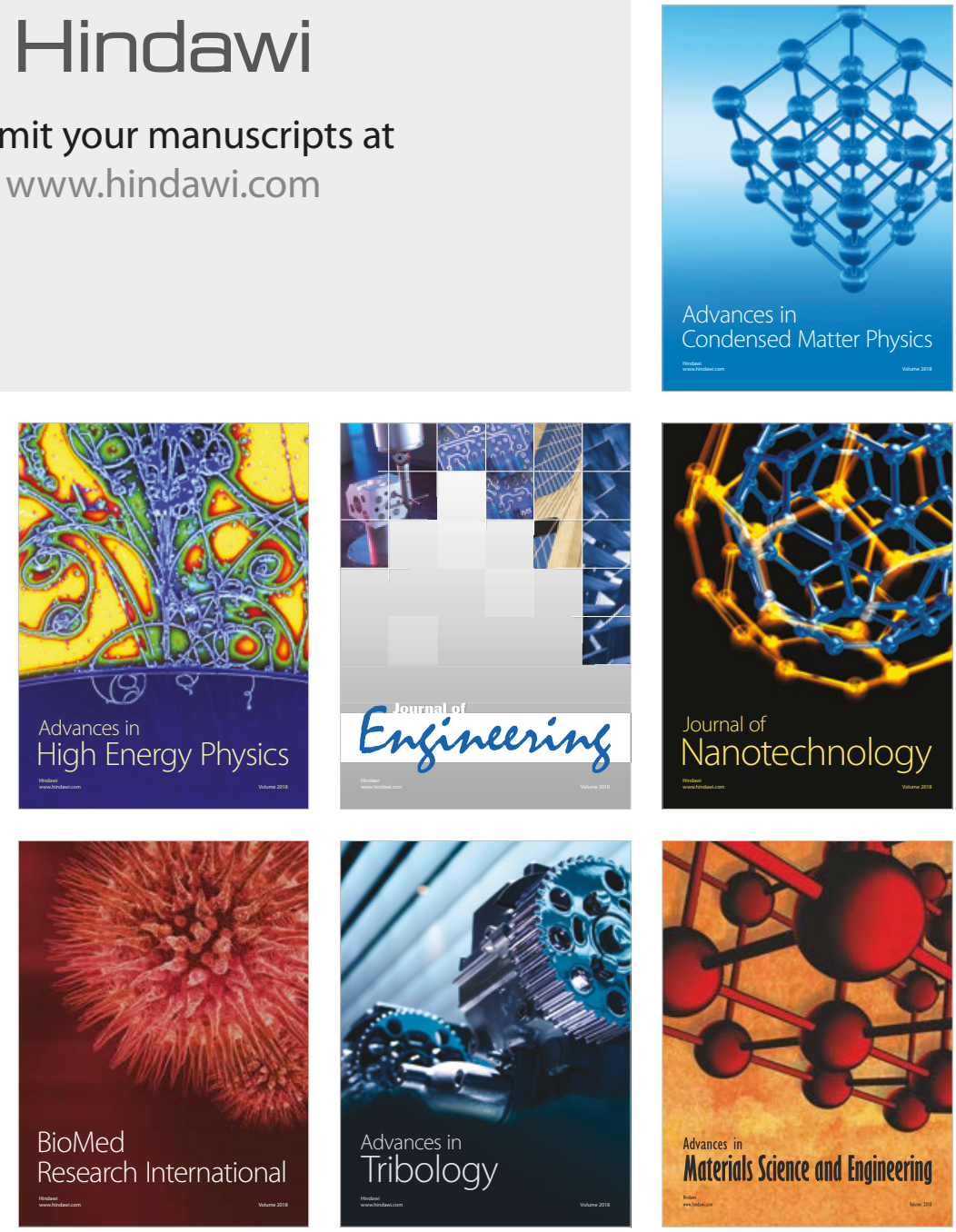